\title{
Ejaculation induced by penile vibratory stimulation in men with spinal cord injuries. The importance of the vibratory amplitude
}

\author{
J Sønksen $\mathrm{MD}^{1}$, F Biering-Sørensen MD $\mathrm{PhD}^{2}$, J K Kristensen MD PhD ${ }^{1}$ \\ ${ }^{1}$ Department of Urology, and ${ }^{2}$ Centre for Spinal Cord Injured, Department TH, \\ Rigshospitalet, The National University Hosptial, Blegdamsvej 9, DK-2100 Copenhagen 1, \\ Denmark
}

\begin{abstract}
A total of 66 men with a spinal cord injury (SCI) and ejaculatory dysfunction were included in two different but comparable study populations I $(n=25)$ and II $(n=41)$. The level of lesion ranged from C2 to L1 (44 complete). Penile vibratory stimulation (PVS) to induce ejaculation was performed with two different types of vibrators in population I and considerably different ejaculation rates (antegrade + retrograde) occurred depending on the vibrator used. Our experience suggested discrepancies between the manufacturers' specifications and the actual vibrator outputs concerning frequencies and peak-to-peak amplitudes. Retrospectively performed determinations revealed that the manufacturers' specifications regarding the frequencies were accurate whereas the peak-to-peak amplitudes were inaccurate. With a frequency of $100 \mathrm{~Hz}$ and determined peak-to-peak amplitudes of $1 \mathrm{~mm}$ and $2.5 \mathrm{~mm}$, ejaculation rates of $32 \%$ and $96 \%$, respectively, were obtained in population I. This indicates that an adequate peak-to-peak amplitude is essential to exceed an 'ejaculatory threshold' in the majority of SCI men. Furthermore, an ejaculation rate of $83 \%$ obtained in a subsequent prospective study of $41 \mathrm{SCI}$ men (population II) verified that a frequency of $100 \mathrm{~Hz}$ and a peak-to-peak amplitude of $2.5 \mathrm{~mm}$ seems to approach the ideal vibrator output. The ejaculation responses obtained by JS (first author) were reproduced when the PVS was performed by the patient or his partner, indicating that the vibrator output is more important than PVS experience. No major adverse reactions due to autonomic dysreflexia were observed. The lowest level of SCI where antegrade or retrograde ejaculation occurred was $T_{9}$ and $L_{1}$, respectively. No absolute predictors for ejeculatory success or failure in relation to patient age, years since lesion, completeness of SCI, urinary bladder management method, hip flexion and bulbocavernous reflexes were observed. Therefore, most SCI men with ejaculatory dysfunction should be considered candidates for PVS.
\end{abstract}

Keywords: spinal cord injuries; vibration; ejaculation; semen; fertility.

\section{Introduction}

It is well known that the vast majority of men with a spinal cord injury (SCI) suffer from ejaculatory dysfunction and if ejaculation is obtained usually they exhibit a very poor semen quality. ${ }^{1}$ The primary cause of infertility in SCI men is their inability to ejaculate. To retrieve sperm from SCI men two methods are primarily used: penile vibratory stimulation (PVS) and rectal electrostimulation. ${ }^{2}$ This study describes the ejaculation response to PVS in men with
SCI related to patient history, level and completeness of the SCI, and reflexes in order to identify possible predictors of ejaculactory success or failure. During the study the importance of the vibratory variables, frequency and, in particular, the amplitude, was evaluated.

\section{Material and methods}

The study protocol was approved by the regional ethical committee, and all patients 
were included only when oral and written informed consent was obtained.

A total of 66 SCI men were included in two different study populations I $(n=25)$ and II $(n=41)$ in order to investigate the possibility of inducing ejaculation by PVS.

\section{Population I}

The first part of this study included 25 consecutive SCI men. The median age was 27 years (range 19-42 years) and median interval from SCI to study entry was 5 years (range 0.6-39 years). The neurological level of cord lesion ranged from $\mathrm{C} 3$ to $\mathrm{L} 1$. Sixteen lesions $(64 \%)$ were complete and nine (36\%) incomplete (Table I).

\section{Population II}

In the second part of this study a different population of 41 consecutive SCI men were included. The median age was 29 years (range 18-44 years) and the median interval from SCI to study entry was 5 years (range $0.2-27$ years). The neurological level of cord lesion ranged from C2 to L1. Twentyeight lesions (68\%) were complete and 13 (32\%) incomplete (Table I).

No statistically significant differences between populations I and II were found with respect to age, years since lesion, level and completeness of SCI (Table I).

\section{Patient history and clinical examinations}

All the SCI men were interviewed regarding their urinary bladder management method, erection capability and ejaculatory function after SCI. Ejaculatory dysfunction was defined as inability to obtain antegrade ejaculation during any kind of sexual activity. Furthermore, the patients were asked whether the urine had been examined for retrograde ejaculation after sexual activity.

The level of SCI was determined as the most caudal, normal functioning spinal cord segment. In complete lesions no sacral sparing was present. The bulbocavernous and hip flexion reflexes were clinically tested in all patients.

\section{Vibrators}

Project development

In 1989 this study was initiated using the only vibrator designed for PVS available in Denmark (vibrator A, see Appendix). The setting of the vibratory variables (frequency and amplitude) were based on the indications reported by Brindley. ${ }^{3,4}$ However, in 1990 a considerably higher ejaculation rate in SCI men was reported ${ }^{5}$ with a different vibrator designed for muscle massage (vibrator B, see Appendix).

As only modest ejaculation rates were obtained with vibrator $\mathrm{A}$ in population I (Table II, vibration procedures $1-3$ ), it was decided to test vibrator B in the same group

Table I Level and completeness of spinal cord lesion in populations I and II

\begin{tabular}{lllr}
\hline \multicolumn{2}{c}{ Spinal cord lesions } & $\begin{array}{c}\text { Population I }(n=25) \\
\text { No. of patients }(\%)\end{array}$ & $\begin{array}{c}\text { Population }(n=41) \\
\text { No. of patients }(\%)\end{array}$ \\
\hline Level & Completeness & & $10(24)$ \\
\hline C2-8 & Complete & $6(24)$ & $7(17)$ \\
& Incomplete & $6(24)$ & $14(34)$ \\
T1-9 & Complete & $7(28)$ & $4(10)$ \\
& Incomplete & $1(4)$ & $4(10)$ \\
T10-L1 & Complete & $3(12)$ & $2(5)$ \\
& Incomplete & $2(8)$ & \\
\hline
\end{tabular}

Completeness within each spinal cord level group between the two populations: $p>0.9$ (Fisher's exact tests).

The three spinal cord level groups, complete and incomplete together, tested between the two populations: $p=0.61\left(\chi^{2}=0.98, \mathrm{df}=2\right)$. 
Table II Results of the vibrations procedures

\begin{tabular}{|c|c|c|c|c|c|c|c|c|c|c|}
\hline \multirow{3}{*}{$\begin{array}{l}\text { Vibration } \\
\text { procedure no. }\end{array}$} & \multirow[t]{3}{*}{$n$} & \multirow{3}{*}{$\begin{array}{l}\text { Type of } \\
\text { vibrator }\end{array}$} & \multirow[t]{3}{*}{ Vibration by } & \multirow{3}{*}{$\begin{array}{l}\text { Frequency } \\
(\mathrm{Hz})\end{array}$} & \multicolumn{2}{|c|}{ Peak-to-peak amplitude (mm) } & \multicolumn{4}{|c|}{ Ejaculation } \\
\hline & & & & & \multirow[t]{2}{*}{ Indicated } & \multirow[t]{2}{*}{ Determined } & \multirow{2}{*}{$\begin{array}{c}\text { Rates }^{a} \\
(95 \% \text { CL })\end{array}$} & \multicolumn{3}{|c|}{ Response } \\
\hline & & & & & & & & A & $\mathrm{R}$ & $N$ \\
\hline 1 & 25 & A & JS & 80 & 2.0 & 1.0 & $32 \%(15-54 \%)$ & 3 & 5 & 17 \\
\hline 2 & 25 & A & JS & 100 & 2.5 & 1.0 & $32 \%(15-54 \%)$ & 3 & 5 & 17 \\
\hline 3 & 25 & A & Patient/partner & 100 & 2.5 & 1.0 & $28 \%(12-49 \%)$ & 2 & 5 & 18 \\
\hline 4 & 25 & $\mathrm{~B}$ & JS & 100 & 1.5 & 2.5 & $96 \%(80-100 \%)$ & 15 & 9 & 1 \\
\hline 5 & 25 & $\mathrm{~B}$ & Patient/partner & 100 & 1.5 & 2.5 & $88 \%(69-97 \%)$ & 13 & 9 & 3 \\
\hline 6 & 22 & $\mathrm{~B}$ & JS & 100 & $-b$ & 1.0 & $32 \%(12-49 \%)$ & 2 & 5 & 15 \\
\hline 7 & 22 & $\mathrm{~B}$ & Patient/partner & 100 & $-b$ & 1.0 & $27 \%(9-45 \%)$ & 2 & 4 & 16 \\
\hline 8 & 41 & $\mathrm{~B}$ & JS & 100 & 1.5 & 2.5 & $83 \%(69-93 \%)$ & 26 & 8 & 7 \\
\hline 9 & 41 & $\mathrm{~B}$ & Patient/partner & 100 & 1.5 & 2.5 & $80 \%(65-91 \%)$ & 26 & 7 & 8 \\
\hline
\end{tabular}

Vibration procedures no. 1-7: population I.

Vibration procedures no. 8-9: population II.

Vibration procedures no. 1-5: the frequencies and amplitudes were determined retrospectively.

Vibration procedures no. 6-9: the frequencies and amplitudes were determined daily before and after vibration.

A: Antegrade; R: Retrograde; $\mathrm{N}$ : None; $\mathrm{CL}=$ confidence limit.

${ }^{a}$ These figures include both antegrade and retrograde ejaculations.

${ }^{b}$ The amplitudes were only indicated for maximum setting (see text).

Comparisons of ejaculation responses:

$p>0.15$ : [ 1 vs 2 ]; [2 vs 3 ]; [ 4 vs 5$]$; [ 6 vs 7 ]; [ 8 vs 9]; [ 2 vs 6 ]; [ 3 vs 7 ]; [4 vs 8 ]; [5 vs 9].

$p \leqslant 0.0001:$ [ 2 vs 4 ]; [ 3 vs 5$]$; [ 6 vs 8 ]; [7 vs 9]. 
of patients which resulted in considerably higher ejaculation rates (Table II, vibration procedures 4 and 5).

Our experience with population I (Table II, vibration procedures 1-5) suggested certain discrepancies between the vibrator specifications indicated by the manufacturers and the actual vibrator outputs. As a consequence the frequencies and amplitudes were determined retrospectively for vibrators $\mathrm{A}$ and $\mathrm{B}$ (see below).

Furthermore, the two vibrators were compared on identical settings in population I which was, however, possible only at the less successful setting of vibrator A (Table II, vibration procedures 2 vs 6 and 3 vs 7).

To be able to evaluate the consistency of the higher ejaculation rates obtained in population I with vibrator B (Table II, vibration procedures 4 and 5), PVS was performed with the same vibrator $B$ in another study population II (Table II, vibration procedures 8 and 9).

\section{Technical specifications}

The two vibrators A and B were electrically driven directly from the net $(220 \mathrm{~V} / 50 \mathrm{~Hz})$.
For vibrator A, a frequency of $0-120 \mathrm{~Hz}$ and a peak-to-peak amplitude of $0-10 \mathrm{~mm}$ was indicated by the manufacturer on two separate scales both being stepless. For vibrator $\mathrm{B}$, the frequency was stationary at $100 \mathrm{~Hz}$ and a peak-to-peak amplitude of $1.5 \mathrm{~mm}$ was indicated by the manufacturer for maximum setting only on a stepless scale. The vibrator knobs of both vibrators were circular with a diameter of $38 \mathrm{~mm}$ and a flat, smooth surface made of hard plastic.

\section{Determination of the vibrator variables frequencies and amplitudes}

The determination of frequencies and peakto-peak amplitudes for vibrators $\mathrm{A}$ and $\mathrm{B}$ were performed after completion of vibration procedures 1-5 (Table II). In the remaining vibration procedures 6-9 (Table II) using vibrator $\mathrm{B}$, the frequencies and peak-to-peak amplitudes were determined daily before and after PVS to ensure the consistency of the vibrator output.

The frequencies were measured with a stroboscope. Equipment to determine the peak-to-peak amplitudes was not available and had to be developed. The equipment

Table III Vibrator specifications and ejaculation rates as reported in the literature

\begin{tabular}{|c|c|c|c|c|}
\hline \multirow[t]{2}{*}{ References } & \multirow{2}{*}{$\begin{array}{l}\text { No. of } \\
\text { patients }\end{array}$} & \multirow{2}{*}{$\begin{array}{c}\text { Ejaculation rates } \\
(\%)\end{array}$} & \multicolumn{2}{|c|}{ Vibration variables } \\
\hline & & & Frequency $(\mathrm{Hz})$ & Amplitude $(\mathrm{mm})$ \\
\hline Whelan $1972^{\mathrm{a}}$ & 63 & $19^{b}$ & $?$ & ? \\
\hline Piera $1973^{14 c}$ & 101 & $27^{\mathrm{b}}$ & $?$ & $?$ \\
\hline Brindley $1981^{3}$ & 21 & $57^{\mathrm{d}}$ & $70-100$ & $1.6-2.4$ \\
\hline Brindley $1984^{4 e}$ & 93 & $53^{\mathrm{d}}$ & 80 & 2.5 \\
\hline Francois et al $1983^{15}$ & 140 & $49 \mathrm{~b}$ & $?$ & $?$ \\
\hline Sakaranti et al 198716 & 33 & $24^{\mathrm{d}}$ & 80 & $1.6-2.4$ \\
\hline Szasz \& Carpenter $1989^{8}$ & 22 & $50^{\mathrm{b}}$ & 120 & $1.0-3.0$ \\
\hline Szasz \& Carpenter $1989^{8}$ & 35 & $34^{\mathrm{d}}$ & $?$ & $?$ \\
\hline Beretta et al $1989^{17}$ & 102 & $71^{\mathrm{d}}$ & 100 & $?$ \\
\hline Beilby \& Keogh $1989^{18}$ & 37 & $51^{\mathrm{b}}$ & $?$ & $2.0-4.0$ \\
\hline Siösteen et al $1990^{5}$ & 32 & $91^{\mathrm{d}}$ & $80-100$ & $1.5-2.5$ \\
\hline Rawicki \& Hill $1991^{19}$ & 9 & $44^{\mathrm{f}}$ & 80 & $?$ \\
\hline Range & & $19-91$ & $70-120$ & $1.0-4.0$ \\
\hline
\end{tabular}

aCited in Reference 13.

bNo distinction between antegrade and/or retrograde ejaculations.

cEjaculation obtained by masturbation or penile vibratory stimulation.

dIncludes both antegrade and retrograde ejaculations.

' The 21 patients from Reference 3 are included.

fOnly antegrade ejaculation reported. 
consists of a voltmeter and an inductive proximity sensor. ${ }^{6}$ The inductive proximity sensor measures the peak-to-peak amplitude which can be read directly in $\mathrm{mm}$ from the scale of a voltmeter. The accuracy of the measurements was within $0.1 \mathrm{~mm}$.

When loading the vibrator knob to the penile surface, the amplitude increases slightly (maximally $0.1 \mathrm{~mm}$ ). The impact of pressure added to the vibrator knob has been estimated and, consequently, taken into account when calculating the determined peak-to-peak amplitudes.

\section{Vibration procedures and observations}

With the patient supine, the prepuce was retracted and the centre of the vibrator knob was placed against the frenulum of the penis. The knob of the vibrator was held in the same position until antegrade ejaculation occurred or for a maximum of $3 \mathrm{~min}$ followed by a pause of $1 \mathrm{~min}$ (one cycle) before the cycle was repeated maximally six times. Number of seconds/minutes from the start of PVS until antegrade ejaculation occurred was noted. Six cycles of 3 min were performed in all patients without antegrade ejaculation. The vibration procedures were performed by JS (first author) and subsequently by the patient himself or his partner, in order to test their own ability to obtain ejaculation.

Ejaculation response was classified as antegrade, retrograde or no ejaculation. Antegrade or retrograde ejaculation was defined as the presence of sperm in the ejaculate or postejaculatory urine. When calculating the ejaculation rates both antegrade and retrograde ejaculations were taken into account. Analyses of the semen quality will be reported on completion of ongoing studies.

Based on previous experience ${ }^{7,8}$ the body reactions, abdominal muscle contractions and leg spasms as response to PVS were clinically observed and registered. Furthermore, the presence or absence of erection during PVS was noted.

\section{Autonomic dysreflexia}

Men with SCI at or above T6 may experience autonomic dysreflexia. ${ }^{9}$ Procedures which involve triggering of ejaculation in SCI men, including PVS, may precipitate an acute episode of autonomic dysreflexia in patients who are susceptible to this syndrome. ${ }^{10,11}$

Before initiating PVS it was checked whether the patients had a history of autonomic dysreflexia, and if any symptoms occurred during PVS the procedure was stopped immediately. In population I no prophylactic medical treatment was given. Based on the experience of Steinberger et $a l^{12}$ it was decided in population II to use nifedipine (10 mg), sublingually, $10 \mathrm{~min}$ before PVS in patients with a history of autonomic dysreflexia and cord lesions at or above T6.

\section{Statistical analyses}

The level and completeness of cord lesion in population I vs II were tested by $\chi^{2}$ test and Fisher's exact test.

For statistical comparisons the ejaculation responses were ranked, i.e. failure: 1 , retrograde: 2, and antegrade: 3; for analysis of unpaired data Mann-Whitney and KruskalWallis rank sum tests were used; for analysis of paired data the expanded McNemar principle was used. When comparing ejaculation rates the sign test was used. Five per cent was chosen as the level of significance.

\section{Results}

Detailed information about the vibration procedures 1-9 including the frequencies and amplitudes used are given in Table II. All amplitudes in the result section are determined peak-to-peak amplitudes.

\section{Determination of vibratory variables}

The frequencies were determined to be in accordance with the specifications from the manufacturers. The determined amplitudes demonstrated that the manufacturers' specifications were inaccurate. Differences between the indicated and determined amplitudes were 1.5 and $1 \mathrm{~mm}$ for vibrators $\mathrm{A}$ and $B$, respectively, at a frequency of $100 \mathrm{~Hz}$ (Table II).

Furthermore, at a frequency of $100 \mathrm{~Hz}$, 
amplitudes of maximally $1.5 \mathrm{~mm}$ and $2.5 \mathrm{~mm}$ could be produced with vibrators $\mathrm{A}$ and $\mathrm{B}$, respectively. Consequently, $\mathrm{B}$ had to be tested on the setting of vibrator $A$ for comparison (vibration procedures 2 vs 6 and 3 vs 7), although it would have been more logical to test vibrator $A$ on the setting of vibrator $\mathrm{B}$ which produced the highest ejaculation rates.

\section{Vibratory variables and ejaculation responses \\ Population I}

When using frequencies of $80 \mathrm{~Hz}$ and $100 \mathrm{~Hz}$, an amplitude of $1 \mathrm{~mm}$ produced identical ejaculation responses (vibration procedures 1 and 2).

A vibrator output of $100 \mathrm{~Hz}$ and an amplitude of $2.5 \mathrm{~mm}$ (vibration procedures 4 and 5) produced significantly higher ejaculation rates $(p<0.0001$, Sign test $)$ than an amplitude of $1 \mathrm{~mm}$ (vibration procedures 2 and 3 ). The higher ejaculation rates at $2.5 \mathrm{~mm}$ were due to converting failures at $1 \mathrm{~mm}$ to successes. When using the same vibrator the ejaculation responses were similar irrespective of whether the investigator (JS) or the patient or his partner performed the procedure.

It was only possible to include 22 of the original 25 patients in the vibration procedures 6 and 7 . When using the same frequency $(100 \mathrm{~Hz})$ and amplitude $(1 \mathrm{~mm})$ the ejaculation responses were similar, irrespective of the vibrator used and who performed the vibrations (vibration procedures 2 and $3 ; 6$ and 7).

\section{Population II}

When prospectively using vibrator $\mathrm{B}$ at a frequency of $100 \mathrm{~Hz}$ and an amplitude of $2.5 \mathrm{~mm}$, the ejaculation responses obtained during vibration procedure 8 and 9 by JS and the patient himself or his partner were similar and demonstrated consistency of replication compared to the results obtained in vibration procedures 4 and 5 .

\section{Evaluation of patient history, observations and ejaculation responses}

This section includes results from both population I and population II. The ejacula- tion responses and clinical observations during PVS reported in this section are based on the results obtained by JS with vibrator B using a frequency of $100 \mathrm{~Hz}$ and an amplitude of $2.5 \mathrm{~mm}$ (vibration procedures 4 and 8 ).

After SCI but prior to PVS none of the patients had had antegrade ejaculation or their urine examined for retrograde ejaculation. Four patients with duration of lesion less than 6 months had not tested their ability to obtain ejaculation post injury.

Ejaculation was obtained in $58(88 \%)$ SCI men. The total number of patients responding to PVS with antegrade or retrograde ejaculations was $41(62 \%)$ and $17(26 \%)$, respectively. Eight (12\%) patients did not obtain ejaculation. During the first cycle of PVS 27 patients responded with antegrade ejaculation within 10-160 s. Between two and six cycles of $3 \mathrm{~min}$ each were required before the remaining 14 patients obtained antegrade ejaculation. No relation was seen between level of cord lesion, completeness of cord lesion, patient age or years since lesion and the stimulation time required to obtain antegrade ejaculation.

The level of cord lesion, urinary bladder management methods, reflexes and clinical observations of body reactions during PVS related to the ejaculation responses are given in the Figs 1-4. The lowest level of cord lesion where antegrade or retrograde ejaculation occurred was T9 and L1, respectively. No relation between ejaculation response and completeness of cord lesion, patient age or years since lesion was seen. All patients with duration of lesion less than 6 months obtained ejaculation (three antegrade: C6, 5 months; T6, 3 months; T8, 2 months; and one retrograde: C6, 3 months).

The general pattern of body reactions in patients with antegrade ejaculation was that continuous abdominal muscle contractions were present at the beginning of PVS but changed to be repetitive contractions when ejaculation occurred. Conversely, leg spasms were initially repetitive but changed to be continuous when ejaculation occurred. In patients with retrograde or no ejaculation from PVS, abdominal muscle contractions and leg spasms, when present at all, were more sporadic, less pronounced and did not 


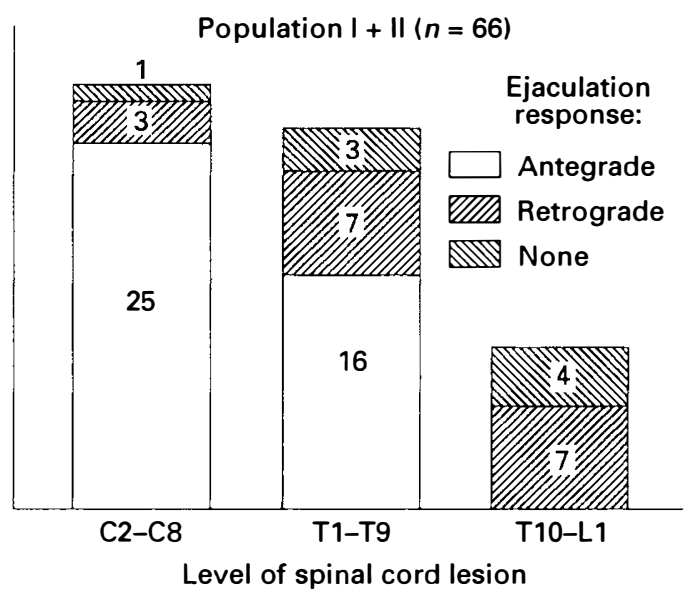

Figure 1 Ejaculation response related to the level of spinal cord lesion: C: Cervical, $\mathrm{T}$ : Thoracic, L: Lumbar. Based on results from vibration procedures 4 and 8 in Table II. Intergroup comparisons: [C2-8] vs [T1-9], $p=0.11$ Mann-Whitney rank sum test); [C2-8] vs [T1-9] vs [T10-L1], $p<0.0001$ (KruskalWallis rank sum test).

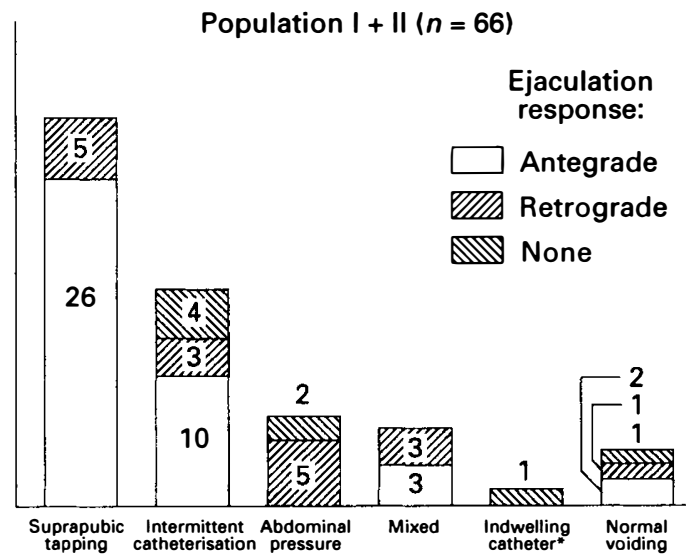

Urinary bladder management methods

Figure 2 Ejaculation response related to urinary bladder management methods. Based on results from vibration procedures 4 and 8 in Table II. Intergroup comparison: [suprapubic tapping] vs [the 5 other groups], $p=0.002$ (MannWhitney rank sum test); [suprapubic tapping] vs [intermittent catherisation] vs [abdominal pressure), $\quad p=0.002$ (Kruskal-Wallis rank sum test).

*The indwelling catheter was removed before penile vibratory stimulation.

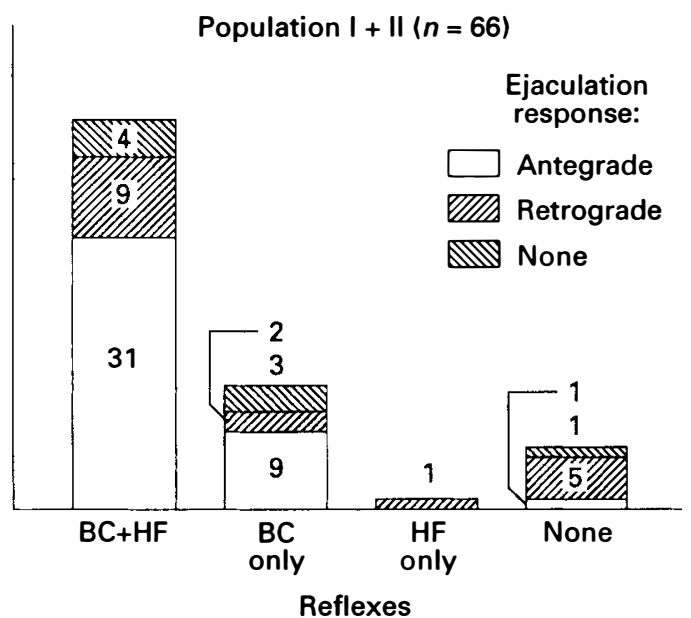

Figure 3 Ejaculation response related to presence/absence of reflexes: BC: Bulbocavernous, HF: Hip flexion. Based on results from vibration procedures 4 and 8 in Table II. Intergroup comparisons: $[\mathrm{BC}+\mathrm{HF}]+[\mathrm{BC}$ only $]$ $+[\mathrm{HF}$ only] vs [none], $p=0.04$ (MannWhitney rank sum test $) ;[\mathrm{BC}+\mathrm{HF}]$ vs $[\mathrm{BC}$ only], $p=0.60$ (Mann-Whitney rank sum test); $[\mathrm{BC}+\mathrm{HF}]$ vs [the three other groups], $p=0.09$ (Mann-Whitney rank sum test).

appear immediately upon stimulation. However, if both abdominal muscle contractions and leg spasms were present during PVS antegrade or retrograde, ejaculation was seen in 46 of 48 patients (Fig 4).

All patients with antegrade $(n=41)$, nine with retrograde $(n=17)$ and six with no ejaculation $(n=8)$ had experienced reflex erection after SCI and prior to PVS. In the group with antegrade ejaculation, erection was seen during the entire PVS in five patients whereas erection appeared only $5-10 \mathrm{~s}$ before ejaculation in 31 patients. In the group with retrograde ejaculation, erection was seen in two patients and only initially during PVS. However, all patients with an erection during PVS obtained either antegrade or retrograde ejaculation (Fig 4).

The clinical presence of both the hip flexion and bulbocavernous reflexes, and at the same time the body reactions, abdominal contractions, leg spasms, and erection during PVS were seen in 27 of 41 patients with antegrade ejaculations. In addition, 19 of the 27 patients had cord lesions at or 


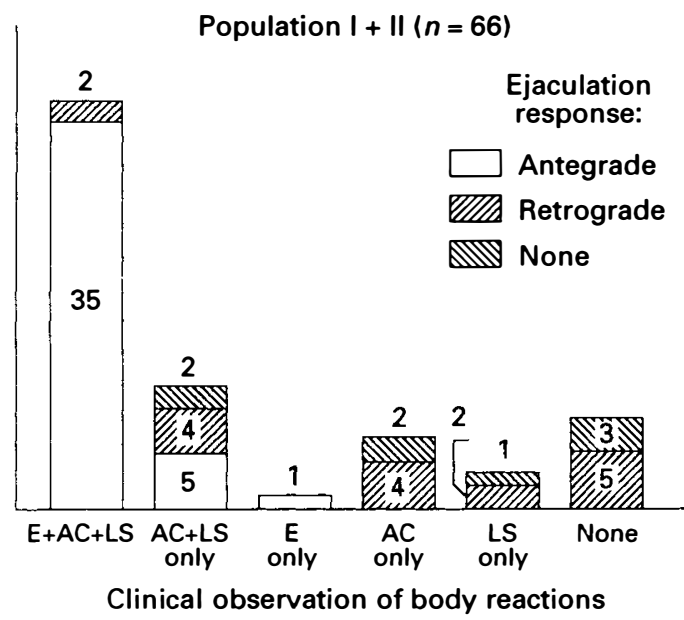

Figure 4 Ejaculation response related to the clinical observations of body reactions during penile vibratory stimulation: erection $(E)$, abdominal muscle contractions (AC), and leg spasms (LS). Based on results from vibration procedures 4 and 8 in Table II. Intergroup comparisons: $[\mathrm{E}+\mathrm{AC}+\mathrm{LS}]$ vs [the 5 other groups], $p<0.00001$ (Mann-Whitney rank sum test); ejaculation response including erection $([\mathrm{E}+\mathrm{AC}+\mathrm{LS}]+[\mathrm{E}])$ compared to ejaculation response without erection ([the four other groups]) revealed $p<0.0001$ (Mann-Whitney rank sum test). Ejaculation response including abdominal muscle contractions and leg spasms $([\mathrm{E}+\mathrm{AS}+\mathrm{LS}]+[\mathrm{AC}+\mathrm{LS}$ only $])$ compared to ejaculation response without both of these body reactions ([the four other groups]) revealed $p<0.0001$ (Mann-Whitney rank sum test).

above T9 and used suprapubic tapping for bladder management. No other considerable overlaps were seen, i.e. the remaining patients were in relation to the above observations distributed within a large variety of combinations as seen from Figs 1-4. Finally, it should be mentioned that in four of 17 patients with retrograde ejaculation the level of cord lesion was at or below T10 and none of the reflexes or body reactions were observed during PVS.

In population I four patients experienced light headache during antegrade ejaculation and PVS was stopped immediately. In no instances did the systolic/diastolic blood pressure exceed $200 / 110 \mathrm{mmHg}$. One pa- tient required treatment with $0.5 \mathrm{mg}$ glycerine nitrate, administrated sublingually, with good effect.

None of the patients in population II experienced discomfort related to autonomic dysreflexia during PVS and ejaculation.

\section{Discussion}

The significant increase in ejaculation rates when using vibrator B instead of vibrator A in study population I was encouraging. Determinations of the peak-to-peak amplitudes proved that the manufacturers' specifications were inaccurate. The frequencies, however, were accurately specified. In study population I, the results (by JS) revealed that a vibrator output of $100 \mathrm{~Hz}$ and a determined peak-to-peak amplitude of $2.5 \mathrm{~mm}$ produced significantly higher ejaculation rates than with a determined peak-topeak amplitude of $1 \mathrm{~mm}(96 \%$ vs $32 \%)$. The fact that the increase in the ejaculation rate originates solely from the patients who failed to ejaculate at the determined peakto-peak amplitude of $1 \mathrm{~mm}$ suggests the presence of an 'ejaculatory threshold'. To obtain high rates of ejaculation by PVS in SCI men, it was necessary to exceed this 'ejaculatory threshold' and our findings suggest that the peak-to-peak amplitude is important in this respect. Whether other frequencies than $100 \mathrm{~Hz}$ at a determined peak-to-peak amplitude of $2.5 \mathrm{~mm}$ would have any impact on the ejaculatory response in SCI men has not been ruled out by this study. In study population II, the obtained ejaculation rate of $83 \%$ (by JS) verified that on a frequency of $100 \mathrm{~Hz}$, a determined peak-to-peak amplitude of $2.5 \mathrm{~mm}$ seems to approach the ideal vibrator output. In addition it proves the consistency of replication in a different study population of SCI men.

The ejaculation rates reported r $^{3-5,8,13-19}$ after PVS in SCI men range from 19 to $91 \%$ (Table III). Comparison of these results is problematic due to methodological inconsistencies. Several different vibrators and settings have been used and it was not possible to extract the optimum vibratory variables (frequency and amplitude) for PVS. In four 
patient groups ${ }^{8,13-15}$ no information was given about the vibratory variables applied. In the other studies ${ }^{3-5,8,16-19}$ differences between indicated frequencies and/or amplitudes and the actual vibrator outputs cannot be ruled out as no information about verification was reported. The considerable differences in the ejaculation rates (Table III) may be explained by unrecognised differences between the indicated vibratory variables and the actual vibrator outputs. Another possible confounding factor may be lack of experience in initiating PVS in SCI men. However, the fact that equivalent ejaculation responses were obtained by JS and the patient or his partner indicates that successful ejaculation depends more on a sufficient vibrator output to exceed an 'ejaculatory threshold' than PVS experience as such.

There was a trend towards a higher number of patients with antegrade ejaculation and a lower number with retrograde or no ejaculation in the cervical group compared to the thoracic group (Fig 1). Completeness of cord lesion, patient age, and years since lesion had no impact on the ejaculation response. Brindley ${ }^{4}$ has reported that PVS usually fails if tried within 6 months of injury. In the present study, however, all patients with duration of lesion less than 6 months obtained ejaculation, which might be because all were out of the spinal shock phase as reflexes were present at the time of PVS.

According to Brindley ${ }^{3,4}$ and Szasz \& Carpenter ${ }^{8}$ the PVS method seems to require an intact spinal cord at the level of T10-S4 in order to induce ejaculation. To some extent our study supports these findings as the lowest levels of cord lesion where antegrade and retrograde ejaculation occurred were T9 and L1, respectively. Despite this trend some patients with cervical, thoracic as well as lumbar lesions did not obtain ejaculation (Fig 1). Four of the seven patients with cord lesions at the T10-L1 level who obtained retrograde ejaculations were clinically evaluated to have complete lesions without sacral sparing. Part of the spinal cord below T10 must still have been intact otherwise ejaculations would not have occurred. ${ }^{3,4,8}$ Whether some degree of in- completeness was present but unrecognised or some patients with complete lesions between $\mathrm{T} 10$ and $\mathrm{L} 1$ are able to obtain retrograde ejaculations cannot be ruled out with certainty by this study. However, ejaculations obtained in SCI men with incomplete lesions as low as the sacral cord level have been reported. ${ }^{17}$ As a consequence all SCI men ought to be considered candidates for PVS since it seems that the clinical examination cannot absolutely exclude that some neurons essential for ejaculatory success are still functioning.

The presence of hip flexion and bulbocavernous reflexes and/or the body reactions abdominal muscle contractions, leg spasms and erection during PVS seem to indicate a higher possibility of antegrade ejaculation. However, even when none of the reflexes and/or body reactions during PVS was present, ejaculation could be obtained in some cases. Brindley ${ }^{4}$ has stated that ejaculation always fails in patients in whom the hips do not flex reflexly when scratching the soles of the feet, since failure of this reflex indicates damage between L2 and S1 segments. In this study (Fig 3), 17 patients obtained ejaculation (10 antegrade and seven retrograde) despite the clinical lack of the hip flexion reflex. Absence of the bulbocavernous reflex indicates damage between S2 and S4. Although it was expected that PVS would fail ${ }^{8}$ seven patients lacking this reflex obtained ejaculation (one antegrade and six retrograde). As previously stated $^{20}$ there is a need for detailed neurophysiological investigations to provide more insight regarding the importance of the presence or absence of the mentioned reflexes. The above findings demonstrate that absolute predictors of ejaculatory success or failure could not be identified among the clinically tested reflexes.

\section{Conclusions}

This study indicates that an adequate peakto-peak amplitude is essential to exceed an 'ejaculatory threshold' to induce ejaculation by PVS in the majority of SCI men. The highest ejaculation rates were obtained with a peak-to-peak amplitude of $2.5 \mathrm{~mm}$ at a 
frequency of $100 \mathrm{~Hz}$. Equal ejaculation responses occurred irrespective of who (JS or patient/partner) performed the PVS.

Antegrade ejaculation with PVS does not commonly occur in SCI men with cord lesion below T9. However, retrograde ejaculations have been observed in SCI men even with sacral cord lesions. In the present study we found no absolute predictors for ejaculatory success or failure. Therefore most SCI men with ejaculatory dysfunction should be considered candidates for PVS.

\section{Acknowledgements}

The authors wish to thank Hans Colstrup MD, Department of Urology, Gentofte Hospital, University of Copenhagen, Denmark for his assistance when this study was designed and initiated. Also thanks to Inger Lauge Jensen
$\mathrm{MD}$ and the staff at the Paraplegic Function, Department of Rheumatology, Viborg Hospital, Denmark for their assistance with inclusion of patients from the western part of Denmark.

\section{Appendix}

Vibrator A: Vibrector, Multicept ApS, Denmark. This is not produced anymore.

Vibrator B: Relax, Nordic Light, Sweden. Further studies have revealed that the amplitude output of this kind of vibrator is not always consistent and adjustments may be required regularly to ensure sufficient vibrator output. However, the vibrator B used in the present study was found to be consistent regarding the vibrator output determined daily before and after PVS in vibration procedures 6-9.

\section{References}

1 Linsenmeyer TA, Perkash I (1991) Review article: Infertility in men with spinal cord injury. Arch Phys Med Rehabil 72: 747-754.

2 Sønksen J, Biering-Sørensen F (1992) Fertility in men with spinal cord or cauda equina lesions. Semin Neurol 12: 106-114.

3 Brindley GS (1981) Reflex ejaculation under vibratory stimulation in paraplegic men. Paraplegia 19: 299-302.

4 Brindley GS (1984) The fertility of men with spinal injuries. Paraplegia 22: 337-348.

5 Siösteen A, Forssman L, Steen Y et al (1990) Quality of semen after repeated ejaculation treatment in spinal cord injury men. Paraplegia 28: 96-104.

6 Brøndum P, Kjeldsen J (1992) Basic measurement principle diagram. Drawn. no. 31S082. Behrendsen Teknik A.S, DK-2860 Søborg, Denmark.

7 Sønksen JOR, Drewes AM, Biering-Sørensen F, Giwercman J (1991) Reflex ejaculation produced by penile vibration in patients with spinal cord lesions. Ugeskr Laeger 153: 2888-2890 (in Danish with summary in English).

8 Szasz G, Carpenter C (1989) Clinical observation in vibratory stimulation of the penis of men with spinal cord injury. Arch Sex Behav 18: 461-474.

9 Rossier AB, Ziegler WH, Duchosal PW, Meylan J (1971) Sexual function and dysreflexia. Paraplegia 9: 51-59.

10 Comarr AE (1985) Sexuality and fertility among spinal cord and/or cauda equina injuries. J Am Paraplegia Soc 8: 67-75.

11 Frankel HL, Mathias CJ (1980) Severe hypertension in patients with high spinal cord lesions undergoing electroejaculation-management with prostaglandin E2. Paraplegia 18: 293-299.

12 Steinberger RE, Ohl DA, Bennett CJ et al (1990) Nifedipine pretreatment for autonomic dysreflexia during electro-ejaculation. Urology 36: 228-231.

13 Tarabulcy E (1972) Sexual function in the normal and in paraplegia. Paraplegia 10: 201-208.

14 Piera JB (1973) The establishment of a prognosis for genito-sexual function in the paraplegic and tetraplegic male. Paraplegia 10: 271-278.

15 Francois N, Jouannet P, Maury M (1983) La fonction génito-sexuelle des paraplégiques. J Urologie 89: 159-166.

16 Sarkarati M, Rossier AB, Fam BA (1987) Experience in vibratory and electroejaculation techniques in spinal cord injury patients: A preliminary report. J Urol 138: 59-63.

17 Beretta G, Chelo E, Zanollo A (1989) Reproductive aspects in spinal cord injured males. Paraplegia 27: 113-118.

18 Beilby JA, Keogh EJ (1989) Spinal cord injuries and anejaculation. Paraplegia 27: 152 (abstract).

19 Rawicki HB, Hill S (1991) Semen retrieval in spinal cord injured men. Paraplegia 29: 443-446.

20 Beckerman H, Becher J, Lankhorst GJ (1993) The effectiveness of vibratory stimulation in anejaculatory men with spinal cord injury. Review article. Paraplegia 31: 689-699. 